\title{
Monique Manoha, et Alexandre Klein (dir.), Objet, bijou et corps : in-corporer
}

Paris, L'Harmattan, 2008. 277 p.

\section{Eugénie Briot}

\section{(2) OpenEdition}

Journals

Édition électronique

URL : http://journals.openedition.org/dht/1612

DOI : 10.4000/dht.1612

ISSN : 1775-4194

Éditeur :

Centre d'histoire des techniques et de l'environnement du Cnam (CDHTE-Cnam), Société des élèves du CDHTE-Cnam

Édition imprimée

Date de publication : 1 décembre 2011

Pagination : 225-226

ISBN : 978-2-9530779-7-1

ISSN : 0417-8726

Référence électronique

Eugénie Briot, « Monique Manoha, et Alexandre Klein (dir.), Objet, bijou et corps : in-corporer »,

Documents pour l'histoire des techniques [En ligne], 20 | 2e semestre 2011, mis en ligne le 13 septembre 2012, consulté le 21 septembre 2020. URL : http://journals.openedition.org/dht/1612 ; DOI : https:// doi.org/10.4000/dht.1612

Ce document a été généré automatiquement le 21 septembre 2020

(c) Tous droits réservés 
Monique Manoha, et Alexandre Klein (dir.), Objet, bijou et corps : in-

\section{corporer}

Paris, L'Harmattan, 2008. 277 p.

\section{Eugénie Briot}

\section{RÉFÉRENCE}

Monique Manoha, et Alexandre Klein (dir.), Objet, bijou et corps : in-corporer, Paris, L'Harmattan, 2008. 277 p. 
1 L'ouvrage intitulé Objet, bijou et corps : incorporer est issu de la quatrième et dernière édition de la Biennale du Bijou

Sous la direction de Monique Manoha et Alexandre Klein Contemporain qui s'est déroulée à Nîmes à l'automne 2005. Il regroupe une vingtaine de communications, avec pour ambition $d$ '«étudier toutes les formes d'incorporation d'objets, formes de l'expérience subjective à chaque fois, qui donnent les "manières d'être ", et qui posent pour un objet - bijou, objetsouvenir, objet-fétiche, etc. - un « vivre avec » souvent ressenti avec nécessité » (p. 6). Conçu dans une perspective pluridisciplinaire, il confronte les analyses de philosophes, de sociologues, d'ethnologues, d'anthropologues, de conservateurs $\mathrm{du}$ patrimoine et $\mathrm{de}$ créateurs, sur des terrains tant européens qu'extra-européens, intéressant des objets

\section{OBJET, BIJOU ET CORPS}

In-corporer historiques (par exemple les bijoux réalisés par les soldats de la première guerre mondiale) aussi bien que contemporains (la moto, le téléphone portable, les robots,...), profanes ou sacrés (amulettes à cordon amérindiennes, reliques,...).

Les travaux historiques et sociologiques consacrés au corps se sont considérablement développés ces dernières années. Bien avant l'histoire des sensibilités, l'histoire des techniques du corps ouverte par les analyses fondatrices de Marcel Mauss étend le champ de la recherche aux techniques appliquées au corps comme aux techniques gestuelles. En plaçant la problématique de l'«in-corporation» au cœur de ses interrogations, l'ouvrage Objet, bijou et corps propose une réflexion à la rencontre des deux champs originellement distingués par Marcel Mauss.

Les contributions de l'ouvrage sont regroupées en cinq parties thématiques : "Corps et expérience corporelle», "Phénomènes d'incorporation», «Bijou - Perspectives anthropologiques", "Bijou - Perspectives historiques», "Bijou - Réflexions contemporaines ». Toutes n'accordent pas une importance comparable aux trois termes « objet », «bijou » et " corps » de l'intitulé, non plus qu'une attention égale au concept $d^{\prime}$ ' in-corporation». L'essentiel de la réflexion, selon les contributions, pourra être porté sur l'un ou l'autre des aspects, sans que les termes de la réflexion soient toujours nécessairement réellement mis en rapport entre eux. Si l'ensemble n'en compose pas moins un tableau fécond, on regrette tout de même l'absence d'une introduction plus significative et développée, qui eut guidé le lecteur pour mettre en perspective les contributions et dresser des ponts entre des objets aussi divers. La première partie "Corps et expérience corporelle», qui ouvre le volume sur trois articles embrassant des thèmes très différents (« Le corps-miroir », de Tito Marques Palmeiro, « Dynamique des représentations socioculturelles $\mathrm{du}$ corps «nu» chez les Zoulgo du NordCameroun », d'Alawadi Zelao et «Corps et radiographie : de l'expérience esthétique de 
l'intimité corporelle » de Margitta Zimmermann), malgré l'intérêt des sujets évoqués, ne vient pas réellement pallier ce manque.

4 Au cœur de la problématique principale, la deuxième partie intitulée "Phénomènes d'incorporation ", qui réunit cinq contributions, est représentative de la richesse des sujets abordés dans l'ouvrage. Dans «Incorporer l'univers domestique ? Esthétique, vie quotidienne et désordre ", Jean Paul Filiod assimile l'action ménagère à une affaire d'incorporation, « entendue ici non comme l'intériorisation de rôles sociaux conformes à des normes culturelles attendues [...] mais comme l'intériorisation d'actions, de gestes, de conduites motrices, connectés à des espaces, des temps, des objets, des êtres, et aux actions d'autres êtres» (p. 58). François Oudin, dans «L'investissement corps/ objet au sein de la "passion de la moto» ", explore pour sa part les paradoxes du corps du motard, soumis à une norme particulière et entraîné dans une "dialectique de la conduite» (p. 63) incarnée par le rapport habileté de l'homme/caractéristiques techniques de la machine, un corps exposé, acrobate, équipé pour la conduite et communiquant, mais demeurant ambivalent, à la fois symbole de puissance et de vulnérabilité. "Incorporer l'affection - A propos des reliques ordinaires contemporaines", de Véronique Dassié, aborde ensuite le sujet des "reliques contemporaines » conservées précieusement par nos contemporains, pour démontrer que si elles relèvent d'une pratique de l'intimité, leur préservation répond à des règles sociales tacites. Pour Corinne Martin, qui traite quant à elle dans sa contribution du " téléphone portable, un objet incorporé ?", le téléphone portable constitue une sorte d'extension de soi, « intégré dans les automatismes et les routines corporelles » (p. 96), souvent personnalisé, et parfois assimilable, grâce à sa miniaturisation, au bijou. Dans "A corps sensible avec les dispositifs techniques : quand l'artefact vient s'investir dans l'humain », enfin, Stéphanie Cardoso démontre que le robot personnel ne prend sens qu'à travers l'usager, tout en permettant à celui-ci d'exister au cœur d'une communauté technophile. "Il se fait complément du corps et des cultures, objet transitionnel redéfinissant le fétiche » (p. 105).

Dans une troisième partie privilégiant une perspective anthropologique, le bijou est envisagé à partir de l'étude de terrains aussi différents que l'Inde ( Inventaire de la dépossession: sur Les Bijoux volés de Premchand (1931)», de Didier Coste), le Népal («Magicité des ornementations corporelles et des parures dans la société Newar Népal», de Sushila Manandhar), l'Amérique du Nord («Les amulettes à cordon amérindiennes, des objets métonymiques des êtres et conceptions du monde», de Marie Goyon), le Mali («Comment «prendre corps»? L'exemple du bébé Soninké » d'Elodie Razy), et le Cameroun (« «Dent d'or» et distinction sociale - Populations du Noun (Ouest Cameroun) » de Caroline Ngamchara Mbouemboue ; « Le collier de Miaffo Tonleu. A propos du don et de l'incorporation dans un groupe de danses rituelles » de Sylvie Perault). Si des rapprochements géographiques sont bien sûr évidents, de réels et fructueux parallèles peuvent également être utilement dressés entre les contributions de Marie Goyon et Elodie Razy.

6 Dans une quatrième partie ancrée dans une perspective historique, Claudette Joannis ("Des cheveux dans un médaillon : mémoire du corps et du cœur ») aborde de front les trois termes du sujet, « objet » « bijou » et « corps ", pour évoquer les bijoux composés de cheveux ou en renfermant, dont la vogue est particulièrement remarquable au XIX ${ }^{\mathrm{e}}$ siècle. Cette contribution offre un écho à celle de Véronique Dassié sur les «reliques ordinaires » intervenant dans la deuxième partie. Dans « Marquer la guerre... Marquer 
sa guerre ", Monique Manoha aborde ensuite la réalisation de bijoux dans les tranchées par les combattants de la première guerre mondiale, où le bijou se fait porteur de valeurs et transforme un objet de mort en message d'espoir. Dans un troisième volet intitulé « American Studio Jewelry Movement - Créateurs de bijoux américains du XX siècle » Ursula Ilse Neumann retrace enfin l'histoire la création de bijoux aux Etats-Unis au XXe siècle.

7 Les «Réflexions contemporaines» qui viennent clore le volume lui offrent une conclusion ouvrant sur des questions tant esthétiques (« «Le bijou est un rien» - Le corps, son ornement et l'histoire du design » d'Annette Geiger et « Vêtement, parure et style de vie » de Catherine Bronnimann) qu'éthiques, comme y invite notamment la contribution de Chloé Dreno (« Du piercing à l'ornement semi-vivant : Incorporer pour repousser les limites »).

\section{AUTEURS}

\section{EUGÉNIE BRIOT}

IRG, Université Paris-Est-Marne-la-Vallée 